\title{
Vulvodynia is not created equally: empirical classification of women with vulvodynia
}

\author{
Meryl Alappattu ${ }^{1,2}$ \\ Georgine Lamvu, \\ Jessica Feranec ${ }^{3,4}$ \\ Kathryn Witzeman ${ }^{5}$ \\ Michael Robinson ${ }^{2,6}$ \\ Andrea Rapkin ${ }^{7}$ \\ 'Department of Physical Therapy, \\ ${ }^{2}$ Center for Pain Research and \\ Behavioral Health, University of \\ Florida, Gainesville, ${ }^{3}$ Division of \\ Surgery, Gynecology Section, Veteran \\ Affairs Medical Center, ${ }^{4}$ University \\ of Central Florida, Orlando, FL, \\ ${ }^{5}$ Women's Integrated Health Program, \\ Department of OBGYN, Denver \\ Health Medical Center, Denver, CO, \\ ${ }^{6}$ Department of Clinical and Health \\ Psychology, University of Florida, \\ Gainesville, FL, ' Department of \\ Obstetrics and Gynecology, David \\ Geffen School of Medicine at the \\ University of California, Los Angeles, \\ CA, USA
}

This article was published in the following Dove Press journal: Journal of Pain Research 6 July 2017

Number of times this article has been viewed
Background: Vulvodynia classification is based on the sensory dimensions of pain and does not include psychological factors associated with the pain experience and treatment outcomes. Previous work has shown that individuals with chronic pain can be classified into subgroups based on pain sensitivity, psychological distress, mood, and symptom severity.

Objective: The aim of this study was to identify distinct subgroups of women with vulvodynia enrolled in the National Vulvodynia Registry. We hypothesized that women with vulvodynia can be clustered into subgroups based on distress and pain sensitivity.

Design: A cross-sectional study.

Methods: We conducted an exploratory hierarchical agglomerative cluster analysis using Ward's cluster method and squared Euclidean distances to identify unique subgroups based on baseline psychological distress and pain sensitivity. The variables included the catastrophizing subscale of the Coping Strategies Questionnaire, the Beck Depression Inventory, the State Trait Anxiety Index-Trait scale, McGill Pain Questionnaire-Affective subscale, and vulvar and pelvic muscle pressure pain sensitivity.

Subjects: Eight sites enrolled women who presented with vaginal or vulval pain of at least 3-month duration.

Results: Two distinct subgroups, high pain sensitivity with high distress $(n=27)$ and low pain sensitivity with low distress $(n=100)$, emerged from the cluster analysis. Validation indicated that subgroups differed in terms of clinical pain intensity, sensory aspects of pain, and intercourse pain. Conclusion: Empirical classification indicates that unique subgroups exist in women with vulvodynia. Providers should be aware of the heterogeneity of this condition with respect to pain-related distress and pain sensitivity.

Keywords: vulvodynia, chronic vulvar pain, pelvic pain, National Vulvodynia Registry, classification, patient subgroups

\section{Background}

Vulvodynia is a pain condition of the vulvar region that affects $16 \%$ of females in the $\mathrm{US}^{1}$ and is defined as pain of at least 3-month duration, without clear identifiable cause, which may have potential associated factors. ${ }^{2}$ Vulvodynia is associated with depression, anxiety, negative impact on quality of life, and limitations in sexual activity. ${ }^{3-5}$ The diagnosis of vulvodynia is based on a detailed history and examination to exclude infectious, dermatologic, neoplastic or hormonal causes, cotton swab testing of vulvar tissues for allodynia vaginal $\mathrm{pH}$, saline and $\mathrm{KOH}$ wet prep, and/or fungal culture. ${ }^{6}$ Current vulvodynia classification is subsequently based on 1) location (generalized or localized), 2) stimulation (provoked pain with contact of the vulva; spontaneous pain in
Correspondence: Meryl Alappattu Department of Physical Therapy, University of Florida, P.O. Box 100154 , Gainesville, FL 32610 0154, USA

Tel +l 352273966 I

Fax +I 3522736109

Email meryl@ufl.edu 
the absence of contact with the vulva, or mixed - provoked and spontaneous), 3) onset (primary - from first genital contact or secondary - after a period of pain-free genital contact), and 4) temporal pattern (intermittent or persistent). ${ }^{2}$

These diagnostic classifications are based predominantly on the sensory dimensions of pain in the vulvar region and does not include emotional and cognitive factors associated with the pain experience, including psychological distress, fear avoidance, ${ }^{7,8}$ catastrophizing, ${ }^{8-10}$ and hypervigilance..$^{8,11}$ Sustained emotional and cognitive distress engenders physiological and biological changes associated with increased health complaints and comorbidities. ${ }^{12,13}$ High levels of cognitive arousal are believed to induce sensitization of pain processing pathways, ${ }^{13,14}$ leading to widespread pain sensitivity and increased clinical pain intensity. Thus, the presence of psychological distress in women with vulvodynia potentially magnifies clinical pain intensity and may also contribute to alterations in central pain processing.

Progress in identifying effective treatments has been slow and current treatments are rarely curative, although emerging work demonstrates that cognitive behavioral therapy is a promising intervention. ${ }^{15-18}$ Still, one potential reason for the lack of progress in identifying effective treatments is that the current definition and evaluation of vulvodynia limit the condition to its sensory components without taking into account the multifactorial pain experience, including emotional factors and pain sensitivity. Pain is a highly personal experience, and inter-individual differences in pain have been widely reported. ${ }^{19-22}$ A well-established expert consensus panel, the International Consultation on Sexual Medicine, recently recommended identifying subgroups of women with vulvodynia to better understand clinical phenotypes to develop personalized treatments. ${ }^{23}$ A large body of converging evidence indicates that individuals with other pain conditions (e.g., fibromyalgia, ${ }^{24-27}$ spinal pain, ${ }^{28,29}$ and whiplash ${ }^{30}$ ) can be classified into subgroups based on factors such as pain sensitivity, psychological distress, mood, symptom severity, and physical functioning. For these other chronic pain conditions, studies suggest that even within seemingly homogenous groups of patients, subgroups of individuals who exhibit different clinical phenotypes exist. Similar heterogeneity may be present in vulvodynia, but current classification of vulvodynia includes only variables related to onset and symptom type.

\section{Objective}

The objective of this study was to identify subgroups of women with vulvodynia using cluster analysis of individuals' psychological distress and pain sensitivity. Cluster analysis is an exploratory data analysis tool used to empirically classify individuals into clusters based on their responses to several variables as suggested by the data. ${ }^{31}$ We hypothesized that women with vulvodynia can be clustered into distinct subgroups based on factors related to distress and pain sensitivity.

\section{Study design}

We conducted these analyses using baseline data from the National Vulvodynia Registry (NVR). ${ }^{32}$ The NVR is a national registry implemented in 2009 at eight different geographical sites across the US and these analyses include NVR participants from 2009 to 2014. The NVR was designed as a prospective cohort study, with enrolled participants completing a comprehensive battery of self-report and clinical assessments at baseline, 6 , and 12 months from baseline. One of the primary objectives of the NVR was to investigate the potential for identifying distinct subtypes of vulvar pain by collecting information from patients on self-reported symptoms, pain levels, physical function, sexual function, and pain-related psychological distress. Institutional review board approval was obtained at all enrollment sites and informed consent was received from all participants. ${ }^{32}$

\section{Subjects}

The primary criterion for entry into the NVR included vaginal or vulvar pain of at least 3 months in duration, severe enough to impair sexual activity and/or quality of life. Participants were screened with a previously validated Harlow's Questionnaire ${ }^{1}$ and clinical examination. Harlow's Questionnaire is a 4-item screening measure that assesses the presence of vulvar pain, its duration, the quality of the pain experienced, and if the symptoms are provoked with contact to the vulvar region. Participants were excluded if they were pregnant, if they were younger than 20 years, or if they were found to have obvious pathology such as acute vaginitis (yeast, bacterial vaginosis, or sexually transmitted infections), dermatoses, neoplasia, or pelvic masses. Women were excluded if they were younger than 20 years because the resources needed to address the following issues were unavailable: 1) asking minors to provide consent; 2) answering sensitive questions related to sexual function, and 3 ) having a parent present during a pelvic examination if the patient is a minor. Women with a history of reproductive tract malignancy, chemotherapy, or radiation therapy were also excluded. ${ }^{32}$

\section{Methods}

After providing written consent, women completed general medical history and psychometric questionnaires on baseline 
characteristics, self-described pain, distress, sexual function, and quality of life. Subsequently, they underwent gynecologic evaluation and pressure sensory testing for assessment of pain sensitivity in the vaginal epithelium and vaginal muscles. The data were collected in a centralized database and de-identified for analysis. Investigators collected all data onto paper forms and all forms were shipped to the Florida Hospital site (lead site). A nosologist performed all data entry. Any pieces of missing data were reconciled with a phone call to the site investigator and subsequent review of medical record.

\section{Outcome measures}

Age, race, and pain duration were collected at baseline via a general medical history questionnaire. Current pelvic pain intensity and quality were measured using the Short-Form McGill Pain Questionnaire sensory subscale (MPQ-S) and a $10 \mathrm{~cm}$ visual analog scale for pain. ${ }^{33,34}$ Average intercourse pain intensity was assessed using the 101-point Numerical Pain Rating Scale by asking participants to report their average pain with intercourse. ${ }^{35,36}$ Lastly, physical functioning was assessed with the physical function domain of the Short-Form $12 .{ }^{37}$

Classification using current terminology related to onset (primary versus secondary) and symptom type (spontaneous versus provoked) was determined by using the following questions for onset type: "Did your pain start before or after first intercourse?" and symptom type: "Have you experienced excessive pain on contact to your genital area? For example, upon insertion of tampon, at time of sexual intercourse, during pelvic examination or when clothing touches that area?" These questions were included as part of the intake paper questionnaire that all participants completed.

The clinical evaluation consisted of a speculum examination, vaginal $\mathrm{pH}$, wet prep, visual inspection, sensory examination of the perineal dermatomes, and palpation of the vaginal muscles. Vaginal mucosa and pelvic muscle-evoked pain sensitivity testing was performed using methods developed by Zolnoun et $\mathrm{al}^{38}$ and have been previously described by Lamvu et al. ${ }^{32}$ Investigators at the eight enrollment sites were trained in the static pressure pain (SPP) procedure by the Principal Investigator of the NVR (GL) to ensure standardization among the investigators prior to formal initiation of the NVR protocol to standardize the testing technique, instrument calibration, and data collection to ensure that all mucosal and muscular data were collected in a comparable manner. All investigators attended one 2-hour training session prior to the start of enrollment and one 2-hour training session after enrollment of the first five participants at his or her respective site. Investigators also attended a yearly meeting with GL to review all testing methodology.

The evoked pain sensitivity measure used in this study was SPP. SPP was applied with a cotton-tipped applicator at five different locations of the upper and lower vulvar vestibule (locations " 2 ", "5", “6", “7”, and "10" as previously described by Zolnoun et $\mathrm{al}^{38}$ ) and digitally at the bilateral levator ani and bulbocavernosus muscles (as previously described by Zolnoun et $\mathrm{al}^{38}$ ) using a single digit with a pressure of $2 \mathrm{~kg}$. Investigators were trained in the application of $2 \mathrm{~kg}$ force at each location as part of the training sessions as already described. Participants were asked to rate their pain from 0 to 10 at each of the five vestibule sites and the three muscular sites when the $2 \mathrm{~kg}$ of pressure was applied with a cotton-tipped applicator (vestibule) and digitally (pelvic floor muscles).

Assessment of psychological distress was conducted using several validated questionnaires:

1) Catastrophizing subscale of the Coping Strategies Questionnaire (CSQ-c): The CSQ-c is a six-item questionnaire that assesses dimensions of helplessness and pessimism. ${ }^{9,39}$ Items are scored from 0 (never do) to 6 (always do that), and the total score ranges from 0 to 36 with higher scores indicative of higher levels of catastrophizing.

2) Beck Depression Inventory-II (BDI): It is a 21-item self-report questionnaire to measure the severity of depression. Scores range from 0 to 63 , with higher scores indicating greater severity of depression. ${ }^{40}$

3) State Trait Anxiety Inventory (STAI): It is a valid, reliable 40 -item measure of state and trait anxiety. Twenty items assess trait anxiety and 20 items measure state anxiety, with scores ranging from 20 to 80 for each scale with higher scores indicating greater levels of anxiety. ${ }^{41}$ For this manuscript, the trait anxiety scale was used.

4) Short-Form McGill Pain Questionnaire affective subscale (MPQ-A): The affective subscale of the McGill Pain Questionnaire is a self-report measure used to describe the emotional experience of pain. ${ }^{42}$

\section{Statistical analysis}

Data were entered into a centralized database and were deidentified prior to analysis. The following variables were included in the cluster analysis: CSQ-c, BDI, STAI-T, MPQA, vestibule composite pain rating, and pelvic floor muscle composite pain rating. To account for the number of evoked pain sensitivity testing sites at the vestibule and pelvic floor muscle (levator ani) musculature, a composite score was 
created for pain ratings at the vestibule and levator ani using the average SPP ratings for the five vestibule sites and three pelvic muscle sites; thus, we calculated one composite score for the vestibule and one composite score for the levator ani. Prior to cluster analysis, all measures were transformed into $z$-scores for standardization.

Analysis was conducted with IBM SPSS Statistics for Windows Version 22 (IBM Corp, Armonk, NY, USA). Descriptive statistics were computed for the sample for demographic characteristics, distress measures, and pain sensitivity. Alpha was set at 0.05 for all analyses.

Exploratory hierarchical agglomerative cluster analysis was conducted using Ward's cluster method and squared Euclidean distances to identify unique subgroups based on distress and pain sensitivity measures. Ward's cluster method was selected as this method aims to join cases into clusters so that the variance within a derived cluster is minimized, while between cluster differences are maximized. ${ }^{43}$ To identify the optimal cluster solution, we examined the resultant dendrogram and agglomeration coefficients. The cluster break point is the change between adjacent coefficients that represents the largest change. A dendrogram is a tree diagram used to illustrate the arrangement of clusters produced by hierarchical clustering. ${ }^{44}$ To validate the cluster analysis, we performed a one-way analysis of variance to examine subgroup differences in clinical pain intensity, intercourse pain, and the MPQ-S scores.

\section{Results}

A total of 327 women participated in the NVR. Of these, 127 women with complete distress and pain sensitivity data at baseline were included in these analyses. Descriptive results for demographic characteristics, distress measures, and pain sensitivity are presented in Table 1 . Two hundred women did not have complete data for our analyses. The reasons for these missing data included the following: patients were enrolled into the NVR but then excluded based on clinical exam findings; patients may have missed questions on the intake questionnaire that was not discovered until review by the nosologist; the physician investigator collecting the data may have missed a piece of information that should have been collected during the clinical examination unintentionally, or due to time restraints in the clinical practice setting. No significant differences existed in age $(p=0.96)$ or duration of symptoms ( $p=0.80$ ) between those included in the following analyses and those excluded due to incomplete data. The distribution of race was also similar between groups.
Table I Demographic and clinical characteristics, distress measures, and pain sensitivity

\begin{tabular}{|c|c|}
\hline Characteristics & $\begin{array}{l}\text { Value, mean (SD); } \\
\text { range }\end{array}$ \\
\hline \multicolumn{2}{|l|}{ Demographic } \\
\hline Age (years) & $34.1(12.0) ; 20-69$ \\
\hline Pain duration (months) & 52.0 (77.4); 0-360 \\
\hline \multicolumn{2}{|l|}{ Race, $N(\%)^{*}$} \\
\hline White & $100(88.5)$ \\
\hline Hispanic & $7(6.2)$ \\
\hline Asian & $2(1.8)$ \\
\hline Native American & $\mathrm{I}(0.9)$ \\
\hline Other & $3(2.7)$ \\
\hline \multicolumn{2}{|l|}{ Measures of distress } \\
\hline $\mathrm{BDI}{ }^{* *}$ & II.I (7.6); 0-38 \\
\hline CSQ-catastrophizing & $14.4(8.4) ; 0-36$ \\
\hline STAI-trait & 43.1 (II.6); 14-72 \\
\hline MPQ-affective & $3.4(3.3) ; 0-12$ \\
\hline \multicolumn{2}{|l|}{ Measures of evoked pain sensitivity } \\
\hline Vestibule SPP rating $\left(x / 10^{* *}\right)$ & $4.4(2.7) ; 0-10$ \\
\hline Vaginal muscle SPP rating $(x / 10 * *)$ & $3.1(2.8) ; 0-10$ \\
\hline \multicolumn{2}{|l|}{ Clinical pain variables } \\
\hline Pain intensity $\left(x / 100^{* * *}\right)$ & 38.2 (32.9); 0-96 \\
\hline MPQ-sensory & $|2.4(6.3) ; 3-3|$ \\
\hline Average intercourse pain & 60.7 (24.4); 0-100 \\
\hline Physical function & $84.7(23.4) ; 0-100$ \\
\hline SF-12 physical function domain & \\
\hline
\end{tabular}

Notes: *Race information was available only for II 3 participants. **Values refer to the II-point numerical pain rating scale. $* * *$ Values refer to the $10 \mathrm{I}$-point numerical pain rating scale. BDI**, clinical cutoff scores for BDI: 0-13 minimal depression, 14-19 mild depression, 20-28 moderate depression, 29-63 severe depression. Abbreviations: BDI, Beck Depression Inventory; CSQ, Coping Strategies Questionnaire; STAl, State Trait Anxiety Inventory; MPQ, McGill Pain Questionnaire; SPP, static pressure pain; SF-12, Short-Form 12.

\section{Cluster analysis}

Pain sensitivity and distress scores were transformed to $z$-scores prior to cluster analysis. Based on the inspection of the dendrogram and agglomeration coefficients, a 2-cluster solution was deemed appropriate. Cluster $1(\mathrm{~N}=100)$ was labeled low distress and low pain sensitivity and Cluster 2 $(\mathrm{N}=27)$ was labeled high distress and high pain sensitivity (Figure 1). Table 2 illustrates the differences between the low distress low pain sensitivity and high distress high sensitivity subgroups. In general, groups differed significantly on variables included in the cluster analysis (pain sensitivity measures and distress measures), in addition to current pelvic pain intensity and intercourse pain intensity. The effect sizes for subgroup differences were generally higher for the psychological measures and evoked pelvic floor muscle pain rating than the evoked vestibule pain rating. No subgroup differences existed in age or duration of pain. The distribution of symptom type and onset into the empirically derived clusters are detailed in Tables 3 and 4 . No differences 


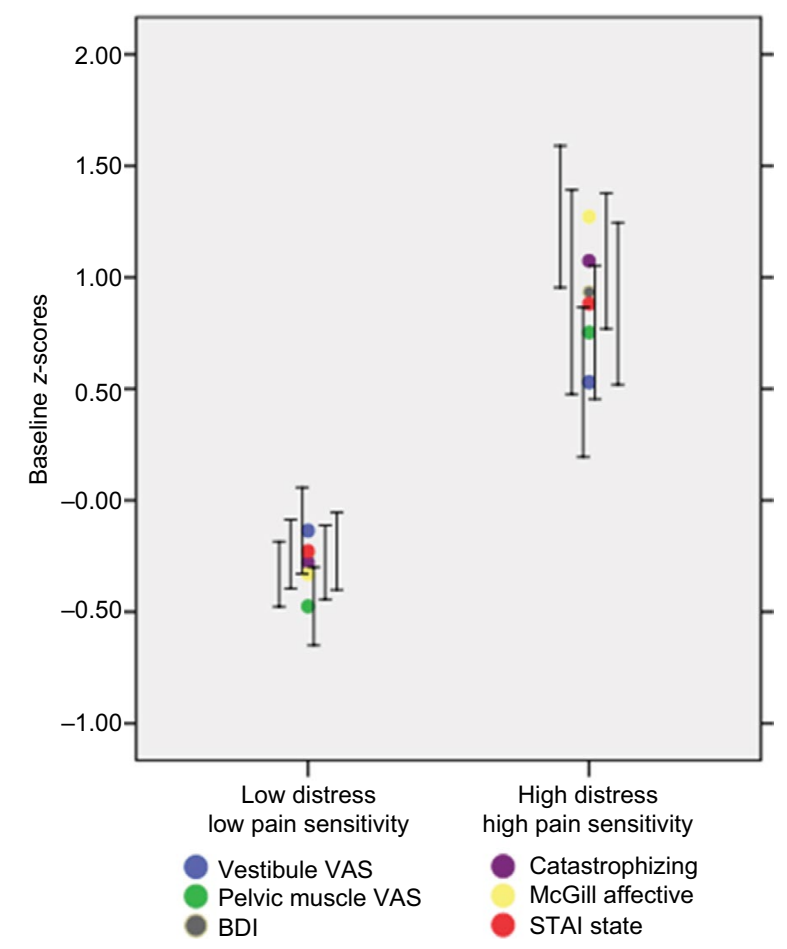

Figure I Cluster subgroups identified based on pain sensitivity and distress measures. Abbreviations: VAS, visual analog scale; BDI, Beck Depression Inventory; STAI, State Trait Anxiety Inventory.

in the proportion of symptom type $\left(X^{2}=0.007, p=0.93\right)$ or onset $\left(X^{2}=0.74, p=0.39\right)$ were observed in the empirically derived clusters.

\section{Discussion}

This cluster analysis of a geographically diverse sample of women with vulvodynia resulted in two unique subgroups. High pain sensitivity and high psychological distress characterized one subgroup and the other was characterized by low pain sensitivity and low psychological distress. Symptom onset (primary vs. secondary) and symptom provocation (provoked vs. spontaneous) did not differ between the two identified subgroups.

The International Society Study of Vulvovaginal Disease, International Society for the Study of Women's Health, and International Pelvic Pain Society developed a pain-based classification system that is useful for the diagnosis of vulvodynia. ${ }^{2,23,45}$ However, the current classification system does not provide a complete phenotype of the individual's pain. Research based on this nomenclature has been fruitful but has not substantially altered or refined management. For example, Brotto et $\mathrm{al}^{46}$ reported that women with secondary provoked vestibulodynia reported more clitoral hood and intercourse pain compared to women with primary provoked vulvodynia. Interestingly, the authors of this study found no psychological differences between the primary and secondary subtypes and concluded that, in general, these subtypes were more similar than they were different in their levels of sexual function, comorbid health conditions, and psychological factors. In a study examining predictors of treatment outcome, Heddini et

Table 2 Subgroup differences on pain sensitivity, distress, and clinical measures

\begin{tabular}{|c|c|c|c|c|}
\hline Measure & $\begin{array}{l}\text { Low distress low sensitivity } \\
(n=100)^{\mathrm{T}}\end{array}$ & $\begin{array}{l}\text { High distress high sensitivity } \\
(n=27)^{\mathrm{T}}\end{array}$ & $p$ & $\begin{array}{l}\text { Effect size } \\
\text { (Cohen's d) }\end{array}$ \\
\hline \multicolumn{5}{|l|}{ Demographic } \\
\hline Age (years) & $34.8(12.3)$ & $31.6(10.5)$ & 0.23 & 0.3 \\
\hline Pain duration (months) & $51.0(74.7)$ & $55.4(87.7)$ & 0.81 & 0.06 \\
\hline \multicolumn{5}{|l|}{ Measures of distress } \\
\hline $\mathrm{BDI} \mathrm{I}^{* * *}$ & $9.2(5.9)$ & $18.2(8.9)$ & $<0.001$ & 1.2 \\
\hline CSQ-catastrophizing & $11.6(7.3)$ & $23.2(6.6)$ & $<0.001$ & 1.7 \\
\hline STAI-trait & $39.0(10.1)$ & $52.0(9.3)$ & $<0.001$ & 1.2 \\
\hline MPQ-affective & $2.3(2.4)$ & $7.7(2.7)$ & $<0.001$ & 2.1 \\
\hline \multicolumn{5}{|c|}{ Measures of evoked pain sensitivity } \\
\hline Vestibule SPP rating $(x / 10)^{*}$ & $4.2(2.6)$ & $5.8(2.3)$ & 0.004 & 0.7 \\
\hline Pelvic muscle SPP rating $(x / 10)^{*}$ & $2.5(2.5)$ & $5.9(2.1)$ & $<0.001$ & 1.5 \\
\hline \multicolumn{5}{|l|}{ Clinical pain variables } \\
\hline VAS current pelvic pain intensity & $35.0(31.6)$ & $50.2(35.4)$ & 0.03 & 0.5 \\
\hline MPQ-sensory & $11.3(5.8)$ & $16.6(6.3)$ & $<0.001$ & 0.9 \\
\hline Average intercourse pain $(x / 100)^{* *}$ & $56.6(25.6)$ & $71.9(16.6)$ & 0.02 & 0.7 \\
\hline \multicolumn{5}{|l|}{ Physical function } \\
\hline SF-I 2 physical function domain & $88.1(20.3)$ & $77.2(30.0)$ & 0.002 & 0.6 \\
\hline
\end{tabular}

Notes: ${ }^{\mathrm{T}}$ Values represent mean (standard deviation). *Values refer to the II-point numerical pain rating scale. **Values refer to the I0I-point numerical pain rating scale. ***Clinical cutoff scores for the BDI: 0-13 minimal depression, 14-19 mild depression, 20-28 moderate depression, 29-63 severe depression.

Abbreviations: BDI, Beck Depression Inventory; CSQ, Coping Strategies Questionnaire; STAI, State Trait Anxiety Inventory; MPQ, McGill Pain Questionnaire; SPP, static pressure pain; VAS, visual analog scale; SF-12, Short-Form 12. 
Table 3 Distribution of onset type into new clusters

\begin{tabular}{|c|c|c|c|}
\hline Onset type & $\begin{array}{l}\text { Low pain sensitivity } \\
\text { low distress }\end{array}$ & $\begin{array}{l}\text { High pain sensitivity } \\
\text { high distress }\end{array}$ & Total \\
\hline Primary & 66 & 22 & 88 \\
\hline Secondary & 16 & 3 & 19 \\
\hline Total & 82 & 25 & 107 \\
\hline
\end{tabular}

Table 4 Distribution of symptom type into new clusters

\begin{tabular}{llll}
\hline $\begin{array}{l}\text { Symptom } \\
\text { type }\end{array}$ & $\begin{array}{l}\text { Low pain sensitivity } \\
\text { low distress }\end{array}$ & $\begin{array}{l}\text { High pain sensitivity } \\
\text { high distress }\end{array}$ & Total \\
\hline Provoked & 58 & 16 & 74 \\
Spontaneous & 28 & 10 & 38 \\
Total & 86 & 26 & 112 \\
\hline
\end{tabular}

al reported that bodily pain was a stronger predictor of success than classification of vestibulodynia as primary or secondary. ${ }^{47}$ Reed et a $1^{48}$ in a large, longitudinal study reported that classifying women by primary or secondary onset did not differentiate women with vulvodynia into empirically derived subgroups, but rather they identified four unique subgroups of vulvodynia using the presence of comorbid pain conditions and the presence of spontaneous vulvar pain. By contrast, the results of the current study indicate that symptom onset and symptom provocation did not differ between our empirically derived subgroups; however, a key difference between the current study and the study by Reed et $\mathrm{a}^{48}$ was that the presence of spontaneous versus provoked pain was used as a variable in their cluster analysis, while the current study examined the proportion of symptom and onset type into our empirically derived clusters post hoc. Additionally, while our sample was heterogeneous, it did not contain equal numbers of women with provoked versus spontaneous vulvodynia and primary versus secondary vulvodynia. Future work in this area that includes a more balanced sample of women with these different subtypes may provide additional clarity about how these groups differ in terms of psychological distress and pain sensitivity.

Classification of any chronic pain condition should be used for diagnostic purposes but more importantly should also help direct treatment. ${ }^{49}$ At present, there are two key issues with the current vulvodynia classification system. First, the diagnosis is based solely on the sensory dimension of pain and time of onset. By definition, pain is a sensory and emotional experience and both of these domains must be evaluated and addressed to obtain a complete picture of the pain experience. ${ }^{50}$ Second, not incorporating psychological variables into the diagnosis may be hampering our ability to adequately manage these patients. Other recent work in this area provides additional support for potential expansion of the diagnosis of vulvodynia. Dargie et $\mathrm{al}^{51}$ developed a vulvar pain questionnaire aimed at evaluating pain severity, emotional and cognitive factors, sexual function, and life and romantic relationship interference. This self-report measure is an important step in potentially improving the diagnosis and management of women with vulvodynia because of its inclusion of factors important to the multidimensional experience of pain.

An abundance of evidence suggests that pain-related psychological distress and sexual dysfunction are higher in women with vulvodynia compared to healthy women. Although psychological treatments are recommended as part of the existing guidelines for vulvodynia, it is unclear how many women with vulvodynia are routinely referred to sexual counselors or clinical psychologists and on what clinical variables the decision is made to provide such referrals. Additionally, while women with vulvodynia report higher psychological distress and sexual dysfunction compared to healthy women, it is still unclear how the presence of psychological distress influences the pain experience, that is, does underlying psychological distress make vulvodynia worse or can the presence of vulvodynia cause psychological distress? This issue needs further study to determine if eliminating or reducing pain intensity will also result in reduction of psychological distress and vice versa.

The presence of pain catastrophizing, pain-related fear, and hypervigilance are negatively associated with pain in a variety of different chronic pain conditions, including vulvodynia, and cognitive treatment approaches have shown promise as potentially effective interventions. A welldesigned trial of interventions for vulvodynia and associated dyspareunia randomized women diagnosed with these conditions to one of three intervention groups: group cognitive behavioral therapy (CBT), EMG intra-vaginal biofeedback, and vestibulectomy. ${ }^{15}$ The CBT intervention included education about vulvodynia and dyspareunia, muscle relaxation, Kegel exercises, and vaginal dilation with the goal of the fear of pain associated with intercourse, increase sexual activity level, and to decrease pain. The findings of this study indicated that all of the interventions resulted in improved sexual function and psychological adjustment outcomes, and these continued to be maintained at 6-month follow-up. As suggested by Bergeron et $\mathrm{al}^{15,18}$ and demonstrated in other studies, ${ }^{52}$ CBT appears to be an effective treatment intervention for women with persistent pelvic pain, particularly vulvodynia. Many of the CBT-related interventions, including activity modification and relaxation training, education about the condition, and self-coping skills, are within the 
scope of clinicians who manage vulvodynia. Additionally, referral to sex therapy may be appropriate to address the negative impact of vulvodynia-related sexual dysfunction on intimate relationships. ${ }^{53,54}$ Intriguingly, a growing body of work also demonstrates that non-psychological interventions are associated with reductions in pain-related psychological distress and improved function. A recent study by Cherkin et al reported that 8 weeks of a mindfulness-based stress reduction, including yoga, for people with chronic low back pain resulted in similar reductions in pain bother and pain-related disability compared to CBT. ${ }^{55}$ This group also reported similar reductions in catastrophizing and improvements in pain self-efficacy when comparing CBT and mindfulness-based stress reduction. ${ }^{56}$ Goldfinger et al reported that in women with vulvodynia randomized to CBT or physical therapy, both groups experienced reductions in pain catastrophizing and perceived control over pain at 6 months but only significant improvements in sexual function were exhibited by the CBT group..$^{57}$

Our work provides the impetus for future work to identify responders of targeted treatments based on their subgroup. Recently published vulvodynia treatment guidelines ${ }^{45}$ recommend that all patients with vulvodynia receive CBT, yet it is unclear which patients with vulvodynia will respond favorably to this type of treatment. Interestingly, the majority of the patients included in our analysis (100 out of 127) fell into the low distress group. When comparing levels of clinical depression with the BDI, the average scores for the low distress group fell into the minimal depression, while the scores in high distress group were consistent with mild depression. Although the NVR was not designed to assess the efficacy of specific treatments, future work in this area should explore if women with vulvodynia who exhibit low distress respond as favorably to psychological therapies as women with vulvodynia who exhibit high distress. We recommend that clinicians who diagnose and manage vulvodynia should screen for psychological distress and appropriately refer to appropriate providers. More research is needed to address how to best incorporate existing work into expanding the classification of vulvodynia to include pain sensitivity and psychological distress. A number of investigators have published work in the assessment of vulvar and pelvic floor muscle pain sensitivity. Pukall et al ${ }^{58}$ developed five vulvalgesiometers to assess pressure pain threshold of the external genital region in women with provoked vulvodynia and control women. They reported that these vulvalgesiometers distinguished between healthy women and those with provoked vulvodynia, and interrater reliability was high. Similarly, Zolnoun et $\mathrm{al}^{38}$ developed both a Q-tipped algometer to assess pressure pain threshold of the vestibule and a thimble algometer to assess pressure pain internally over the pelvic floor muscles. Both devices demonstrated good intrarater, interrater, and test-retest reliability. Collectively, these studies demonstrate that assessment of mucosa and pelvic floor muscles may be performed in a reliable manner and may correctly distinguish between women with vulvodynia and healthy women. However, both of these studies comprised small samples and more research into standardized pain sensitivity assessment as part of routine clinical practice is warranted. Additionally, future work should investigate if an expanded classification system will help to personalize care and improve patient outcomes related to pain, sexual function, and psychological distress.

\section{Limitations}

These findings may not be representative of all women with vulvodynia. Only women who sought care for vulvodynia and consented to participate in the NVR were included and only one-third of the women enrolled in the NVR were included in the cluster analysis because those excluded did not complete clinical exam and/or self-report questionnaire data. Additionally, our data are based on a single time point, and factors that may influence pain sensitivity, including phase of the menstrual cycle, were not recorded and thus not controlled for in these analyses. When assessing for the presence of provoked versus spontaneous symptoms, the question we used was related to the presence of provoked pain symptoms but did not specifically ask spontaneous pain with provoked symptoms. The variables in the cluster analysis were included based on our hypotheses regarding pain sensitivity and psychological distress; inclusion of other variables into the analysis may have reduced or increased number and/or type of clusters. Lastly, while our sample consisted of data from eight different geographical sites, our sample was overwhelmingly White ( $88.5 \%$ of sample) and the presence of other races was slightly lower than a recently published study on the racial distributions of vulvodynia. ${ }^{59}$ Thus, our sample may not be fully representative of the vulvodynia population.

\section{Conclusion}

The presence of two distinct, empirically derived subgroups from our cluster analysis of a sample of women with vulvodynia suggests that the heterogeneity of vulvodynia extends beyond temporal onset and provocation of symptoms. The diagnostic classification of vulvodynia should include psychological distress in addition to the existing classifiers of 
temporal onset and symptom provocation. These results also support screening for psychological distress and referrals to appropriately trained providers.

\section{Acknowledgments}

This research was supported by funding from the National Vulvodynia Association and the Patty Brisben Foundation for Women's Sexual Health and Florida Hospital (Orlando, FL, USA). Methodology developed for the NVR was developed with the help of Dr. Denniz Zolnoun with funding from the National Institutes of Health, K23 HD053631-01: Refining Diagnosis Criteria of Pain Disorder: Vulvar Vestibulitis Syndrome. Dr. Alappattu is supported by the Rehabilitation Research Career Development Program (National Center for Medical and Rehabilitation Research and National Institute for Neurological Disorders and Stroke K12 HD055929).

\section{Disclosure}

Dr Jessica Feranec serves on the speaker's bureau for Exparel® (bupivacaine liposome injectable suspension) produced by Pacira Pharmaceutical Inc. Dr Lamvu serves on the Advisory Board for Daiichi Sankyo Pharmaceuticals and has research funding from Pfizer Grants for Learning and Change. The other authors report no conflicts of interest in this work.

\section{References}

1. Harlow BL, Stewart EG. A population-based assessment of chronic unexplained vulvar pain: have we underestimated the prevalence of vulvodynia? J Am Med Womens Assoc (1972). 2003;58(2):82-88.

2. Bornstein J, Goldstein AT, Stockdale CK, et al. 2015 ISSVD, ISSWSH, and IPPS consensus terminology and classification of persistent vulvar pain and vulvodynia. $J$ Sex Med. 2016;13(4):607-612.

3. Masheb RM, Wang E, Lozano C, Kerns RD. Prevalence and correlates of depression in treatment-seeking women with vulvodynia. J Obstet Gynaecol. 2005;25(8):786-791.

4. Aerts L, Bergeron S, Corsini-Munt S, Steben M, Pâquet M. Are primary and secondary provoked vestibulodynia two different entities? A comparison of pain, psychosocial, and sexual characteristics. $J$ Sex Med. 2015;12(6):1463-1473.

5. Arnold LD, Bachmann GA, Rosen R, Kelly S, Rhoads GG. Vulvodynia: characteristics and associations with comorbidities and quality of life. Obstet Gynecol. 2006;107(3):617-624.

6. Stockdale CK, Lawson HW. 2013 vulvodynia guideline update. J Low Genit Tract Dis. 2014;18(2):93-100.

7. Desrochers G, Bergeron S, Khalifé S, Dupuis MJ, Jodoin M. Provoked vestibulodynia: psychological predictors of topical and cognitivebehavioral treatment outcome. Behav Res Ther. 2010;48(2):106-115.

8. Desrochers G, Bergeron S, Khalifé S, Dupuis MJ, Jodoin M. Fear avoidance and self-efficacy in relation to pain and sexual impairment in women with provoked vestibulodynia. Clin J Pain. 2009;25(6):520-527.

9. Quartana PJ, Campbell CM, Edwards RR. Pain catastrophizing: a critical review. Expert Rev Neurother. 2009;9(5):745-758.

10. Pukall CF, Binik YM, Khalifé S, Amsel R, Abbott FV. Vestibular tactile and pain thresholds in women with vulvar vestibulitis syndrome. Pain. 2002;96(1-2):163-175.
11. Payne KA, Binik YM, Amsel R, Khalifé S. When sex hurts, anxiety and fear orient attention towards pain. Eur J Pain. 2005;9(4):427-436.

12. Ursin H, Eriksen HR. Sensitization, subjective health complaints, and sustained arousal. Ann N Y Acad Sci. 2001;933:119-129.

13. Ursin H, Eriksen HR. The cognitive activation theory of stress. Psychoneuroendocrinology. 2004;29(5):567-592.

14. Brosschot JF. Cognitive-emotional sensitization and somatic health complaints. Scand J Psychol. 2002;43(2):113-121.

15. Bergeron S, Binik YM, Khalifé S, et al. A randomized comparison of group cognitive--behavioral therapy, surface electromyographic biofeedback, and vestibulectomy in the treatment of dyspareunia resulting from vulvar vestibulitis. Pain. 2001;91(3):297-306

16. Bergeron S, Khalifé S, Glazer HI, Binik YM. Surgical and behavioral treatments for vestibulodynia: two-and-one-half year follow-up and predictors of outcome. Obstet Gynecol. 2008;111(1):159-166.

17. Corsini-Munt S, Bergeron S, Rosen NO, Mayrand MH, Delisle I. Feasibility and preliminary effectiveness of a novel cognitive-behavioral couple therapy for provoked vestibulodynia: a pilot study. J Sex Med. 2014;11(10):2515-2527.

18. Bergeron S, Lord MJ. The integration of pelvi-perineal re-education and cognitive-behavioural therapy in the multidisciplinary treatment of the sexual pain disorders. Sex Relation Ther. 2003;18(2): $135-141$.

19. Fillingim RB. Individual differences in pain responses. Curr Rheumatol Rep. 2005;7(5):342-347.

20. Coghill RC. Individual differences in the subjective experience of pain: new insights into mechanisms and models. Headache. 2010;50(9):1531-1535.

21. Vachon-Presseau E, Martel MO, Roy M, et al. Acute stress contributes to individual differences in pain and pain-related brain activity in healthy and chronic pain patients. $J$ Neurosci. 2013;33(16):6826-6833.

22. Nielsen CS, Staud R, Price DD. Individual differences in pain sensitivity: measurement, causation, and consequences. $J$ Pain. 2009;10(3):231-237.

23. Pukall CF, Goldstein AT, Bergeron S, et al. Vulvodynia: definition, prevalence, impact, and pathophysiological factors. J Sex Med. 2016;13(3):291-304.

24. Giesecke T, Williams DA, Harris RE, et al. Subgrouping of fibromyalgia patients on the basis of pressure-pain thresholds and psychological factors. Arthritis Rheum. 2003;48(10):2916-2922.

25. Docampo E, Collado A, Escaramís G, et al. Cluster analysis of clinical data identifies fibromyalgia subgroups. PLoS One. 2013;8(9): e74873.

26. Turk DC, Okifuji A, Sinclair JD, Starz TW. Pain, disability, and physical functioning in subgroups of patients with fibromyalgia. J Rheumatol. 1996;23(7):1255-1262.

27. Wilson HD, Robinson JP, Turk DC. Toward the identification of symptom patterns in people with fibromyalgia. Arthritis Rheum. 2009;61(4):527-534.

28. Beneciuk JM, Robinson ME, George SZ. Low back pain subgroups using fear-avoidance model measures: results of a cluster analysis. Clin J Pain. 2012;28(8):658-666.

29. Coronado RA, Bialosky JE, Robinson ME, George SZ. Pain sensitivity subgroups in individuals with spine pain: potential relevance to shortterm clinical outcome. Phys Ther. 2014;94(8):1111-1122.

30. Pedler A, Sterling M. Patients with chronic whiplash can be subgrouped on the basis of symptoms of sensory hypersensitivity and posttraumatic stress. Pain. 2013;154(9):1640-1648.

31. Romesburg HC. Cluster Analysis For Researchers. Morrisville, NC: Lulu Press; 2004.

32. Lamvu G, Nguyen RH, Burrows LJ, et al. The evidence-based vulvodynia assessment project. A national registry for the study of vulvodynia. J Reprod Med. 2015;60(5-6):223-235.

33. Grafton KV, Foster NE, Wright CC. Test-retest reliability of the shortform McGill pain questionnaire: assessment of intraclass correlation coefficients and limits of agreement in patients with osteoarthritis. Clin J Pain. 2005;21(1):73-82. 
34. Wright KD, Asmundson GJ, McCreary DR. Factorial validity of the short-form McGill pain questionnaire (SF-MPQ). Eur J Pain. 2001;5(3):279-284.

35. Jensen MP, Karoly P, Braver S. The measurement of clinical pain intensity: a comparison of six methods. Pain. 1986;27(1):117-126.

36. Jensen MP, Turner JA, Romano JM. What is the maximum number of levels needed in pain intensity measurement? Pain. 1994;58(3): 387-392.

37. Busija L, Pausenberger E, Haines TP, Haymes S, Buchbinder R, Osborne RH. Adult measures of general health and health-related quality of life: medical outcomes study short form 36-item (SF-36) and short form 12-item (SF-12) health surveys, Nottingham health profile (NHP), sickness impact profile (SIP), medical outcomes study short form 6D (SF-6D), health utilities index mark 3 (HUI3), quality of well-being scale (QWB), and assessment of quality of life (AQoL). Arthritis Care Res (Hoboken). 2011;63 Suppl 11:S383-S412.

38. Zolnoun D, Bair E, Essick G, Gracely R, Goyal V, Maixner W. Reliability and reproducibility of novel methodology for assessment of pressure pain sensitivity in pelvis. J Pain. 2012;13(9):910-920.

39. Rosenstiel AK, Keefe FJ. The use of coping strategies in chronic low back pain patients: relationship to patient characteristics and current adjustment. Pain. 1983;17(1):33-44.

40. Beck AT, Steer RA, Ball R, Ranieri W. Comparison of Beck depression inventories -IA and -II in psychiatric outpatients. J Pers Assess. 1996;67(3):588-597.

41. Spielberger CD. Manual for the State-Trait Anxiety Inventory (Form Y). Palo Alto, CA: Mind Garden; 1983.

42. Lovejoy TI, Turk DC, Morasco BJ. Evaluation of the psychometric properties of the revised short-form McGill pain questionnaire. J Pain 2012;13(12):1250-1257.

43. Ward Jr JH. Hierarchical grouping to optimize an objective function. J Am Stat Assoc. 1963;58(301):236-244.

44. Caliński T, Harabasz J. A dendrite method for cluster analysis. Commun Stat. 1974;3(1):1-27.

45. Goldstein AT, Pukall CF, Brown C, Bergeron S, Stein A, Kellogg-Spadt S. Vulvodynia: assessment and treatment. J Sex Med. 2016;13(4):572-590.

46. Brotto LA, Sadownik LA, Thomson S, et al. A comparison of demographic and psychosexual characteristics of women with primary versus secondary provoked vestibulodynia. Clin J Pain. 2014;30(5): $428-435$.
47. Heddini U, Bohm-Starke N, Nilsson KW, Johannesson U. Provoked vestibulodynia--medical factors and comorbidity associated with treatment outcome. J Sex Med. 2012;9(5):1400-1406.

48. Reed BD, Plegue MA, Williams DA, Sen A. Presence of spontaneous pain and comorbid pain conditions identifies vulvodynia subgroups. $J$ Low Genit Tract Dis. 2016;20(1):57-63.

49. Dworkin RH, Bruehl S, Fillingim RB, Loeser JD, Terman GW, Turk DC. Multidimensional diagnostic criteria for chronic pain: introduction to the ACTTION-American pain society pain taxonomy (AAPT). J Pain 2016;17(9 Suppl):T1-T9.

50. Turk DC, Fillingim RB, Ohrbach R, Patel KV. Assessment of psychosocial and functional impact of chronic pain. J Pain. 2016;17(9 Suppl):T21-T49.

51. Dargie E, Holden RR, Pukall CF. The vulvar pain assessment questionnaire inventory. Pain. 2016;157(12):2672-2686.

52. Masheb RM, Kerns RD, Lozano C, Minkin MJ, Richman S. A randomized clinical trial for women with vulvodynia: cognitive-behavioral therapy vs. supportive psychotherapy. Pain. 2009;141(1-2):31-40.

53. Rosenbaum TY. Musculoskeletal pain and sexual function in women. J Sex Med. 2009;7(2 Pt 1):645-653.

54. Rosenbaum TY. Physiotherapy treatment of sexual pain disorders. $J$ Sex Marital Ther. 2005;31(4):329-340.

55. Cherkin DC, Sherman KJ, Balderson BH, et al. Effect of mindfulnessbased stress reduction vs cognitive behavioral therapy or usual care on back pain and functional limitations in adults with chronic low back pain: a randomized clinical trial. JAMA. 2016;315(12):1240-1249.

56. Turner JA, Anderson ML, Balderson BH, Cook AJ, Sherman KJ, Cherkin DC. Mindfulness-based stress reduction and cognitive behaviora therapy for chronic low back pain: similar effects on mindfulness, catastrophizing, self-efficacy, and acceptance in a randomized controlled trial. Pain. 2016;157(11):2434-2444.

57. Goldfinger C, Pukall CF, Thibault-Gagnon S, McLean L, Chamberlain S. Effectiveness of cognitive-behavioral therapy and physical therapy for provoked vestibulodynia: a randomized pilot study. J Sex Med. 2016;13(1): 88-94.

58. Pukall CF, Young RA, Roberts MJ, Sutton KS, Smith KB. The vulvalgesiometer as a device to measure genital pressure-pain threshold. Physiol Meas. 2007;28(12):1543-1550.

59. Reed BD, Legocki LJ, Plegue MA, Sen A, Haefner HK, Harlow SD. Factors associated with vulvodynia incidence. Obstet Gynecol. 2014;123(2 Pt 1):225-231.
Journal of Pain Research

\section{Publish your work in this journal}

The Journal of Pain Research is an international, peer reviewed, open access, online journal that welcomes laboratory and clinical findings in the fields of pain research and the prevention and management of pain. Original research, reviews, symposium reports, hypothesis formation and commentaries are all considered for publication

\section{Dovepress}

The manuscript management system is completely online and includes a very quick and fair peer-review system, which is all easy to use. Visit http://www.dovepress.com/testimonials.php to read real quotes from published authors. 